\title{
State-of-the-art of Metamaterials: Characterization, Realization and Applications
}

\author{
Giuseppe Ruvio ${ }^{1,2}$ \& Giovanni Leone ${ }^{1}$ \\ ${ }^{1}$ Seconda Università di Napoli, Dipartimento di Ingegneria Industriale e dell'Informazione, Via Roma 29, 81031 Aversa \\ (CE), Italy \\ ${ }^{2}$ Dublin Institute of Technology, Antenna \& High Frequency Research Centre, Kevin Street, Dublin 8, Ireland \\ Correspondence: Giuseppe Ruvio, Dublin Institute of Technology, Antenna \& High Frequency Research Centre, Kevin \\ Street, Dublin 8, Ireland
}

Received: May 10, 2014 Accepted: May 29, 2014 Available online: July 2, 2014

doi:10.11114/set.v1i2.456

URL: http://dx.doi.org/10.11114/set.v1i2.456

\begin{abstract}
Metamaterials is a large family of microwave structures that produces interesting $\varepsilon$ and $\mu$ conditions with huge implications for numerous electromagnetic applications. Following a description of modern techniques to realize epsilon-negative, mu-negative and double-negative metamaterials, this paper explores recent literature on the use of metamaterials in hot research areas such as metamaterial-inspired microwave components, antenna applications and imaging. This contribution is meant to provide an updated overview of complex microwave engineering for the generation of different types of metamaterials and their application in topical electromagnetic scenarios.
\end{abstract}

Keywords: Metamaterial, Epsilon-Negative Materials, Mu-Negative Materials, Double-Negative Materials

\section{Introduction}

In 1968, Victor Veselago investigated (Veselago, 1968) the electromagnetic properties of a hypothetical material with both negative electric permittivity and magnetic. This material does not exist in nature, but its study unveiled numerous new electromagnetic problems and perspectives. In Veselago's description, materials with negative permittivity and negative permeability had the very basic property that the index of refraction is negative. Also other researchers investigated the electromagnetic behavior associated with negative refraction (Mandelshtam, 1950; Sivukhin, 1957; Silin, 1959), but Veselago anticipated that materials with negative index would lead to revolutionary implications for the interaction between wave and matter that change geometric and wave optics perspectives.

Since electromagnetic waves do not interact with each other, materials must be used to control and manipulate them by leveraging coupling fields and charges. Any improvement in achieving desired material conditions corresponds to an advanced control of electromagnetic waves. The diversity in the exploration of metamaterials shows how profound this concept is and how their analysis and design can impact many different application areas in electromagnetics. This research has recently advanced in both the characterization of materials and their application in complex transmission lines. Moreover, metamaterials based on engineered surfaces are particularly interesting since losses become under control and have been considered from both fundamental and applied perspectives. Finally, metamaterial antennas and metamaterial elements that enhance antenna performance still remain a strong topic of interest and a fruitful area of research. Fig. 1 summarizes recent research on metamaterials.

This paper is organized as follows: Section 2 gives a broad overview of the most innovative realization techniques of epsilon-, mu- and double-negative metamaterials; the following sections discuss most important applications that have been suggested in the literature regarding microwave components, antennas and imaging; a short section on fabrication challenges and conclusions end the paper.

\section{Realization of Epsilon-Negative, Mu-Negative and Double-Negative Metamaterial}

Metamaterials are artificially constructed structures that present unusual electrical properties such as negative (or near-zero) values of effective permeability and/or effective permittivity over a certain narrow frequency band. The conventional split-ring resonator (SRR) is a regular sub-wavelength unit cell module that was introduced (Pendry, Holden, Robbins \& Stewart, 1999; Smith, Padilla, Vier, Nemat-Nasser \& Schultz, 2000; Tao, Padilla, Zhang \& Averitt, 
2011) to build periodic arrays which can be excited magnetically or electrically to obtain a $\mu$-negative (MNG) or an $\varepsilon$-negative (ENG) region, respectively, above its LC-type resonance frequency. An overview of SRR geometries is given in Fig. 2. A Double Negative metamaterial, DNG, takes its definition from both the real part of the overall effective permittivity, $\varepsilon_{r e}$, and the real part of the magnetic permeability, $\mu_{r e}{ }^{\prime}$, being simultaneously negative. This characteristic leads to the propagation of a "backward wave" in which the phase velocity and group velocity of the wave have opposite signs. The direction of the phase velocity is opposite to that of the energy flow. As explained by Hand and Cummer (Hand \& Cummer, 2009), in current realizations of DNG materials, $\varepsilon_{r}{ }^{\prime}<0$ is commonly provided by a metallic element that resonates near the plasma frequency, and the $\mu_{r}^{\prime}<0$ condition is given by a split-ring resonator. These elements are then arranged periodically with the overall result of a highly anisotropic material that exhibits DNG behavior for electromagnetic radiation that is incident on the structure over a narrow range of angles. On the other hand, it was shown theoretically by Holloway et al. (Halloway, Kuester Baker-Jarvis \& Kabos, 2003) that a composite medium consisting of non-metallic, magneto-dielectric spherical particles embedded in a matrix can exhibit DNG behavior for wavelengths where resonance occurs. This work is based on the theory developed by Lewin (Lewin, 1947), in which a low-order solution according to Mie scattering theory is obtained for a plane wave incident on a simple cubic array of magneto-dielectric spheres. It is interesting to observe that although the theoretical development relies on the simple cubic lattice arrangement of particles, the dipolar treatment of the particle polarizations is independent of microstructure and this still holds for a random dispersion of spheres in a matrix, provided that the particles are well-separated.

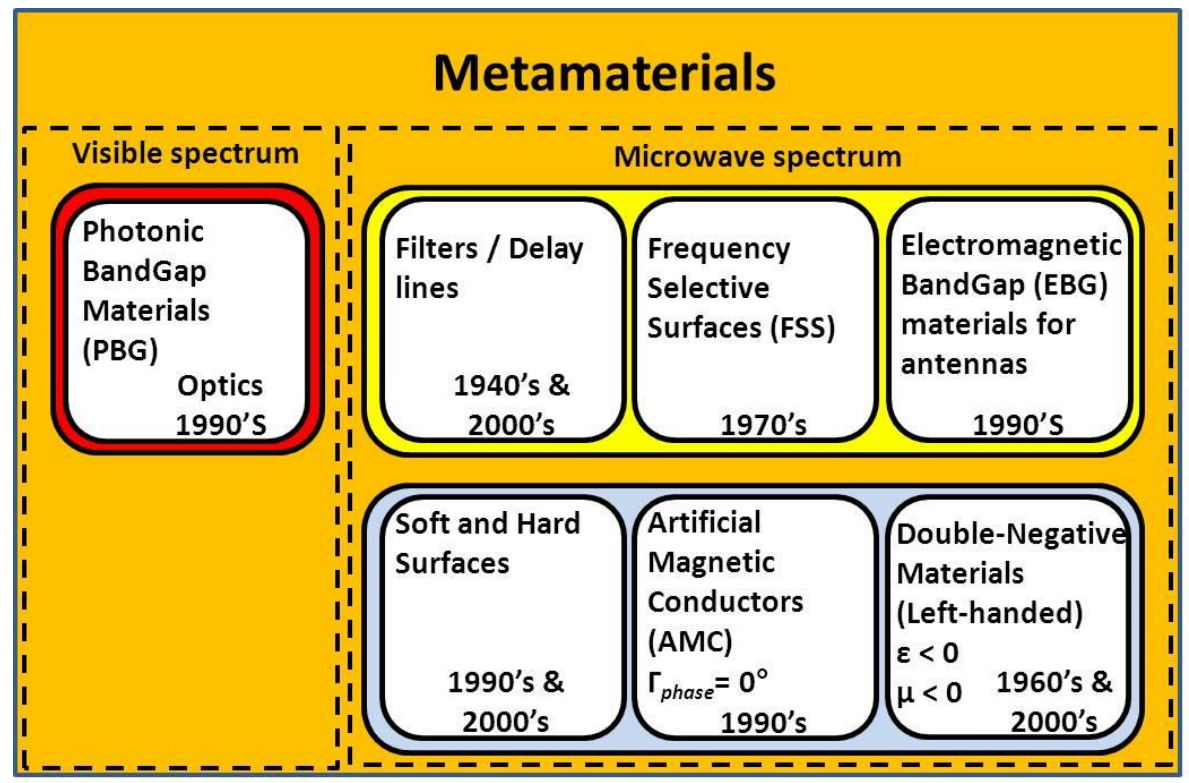

Figure 1. Overview of metamaterials in recent history.

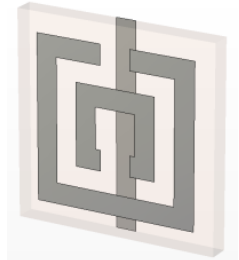

Edge-coupled SRR

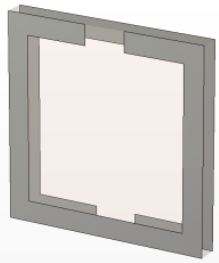

Broadside SRR

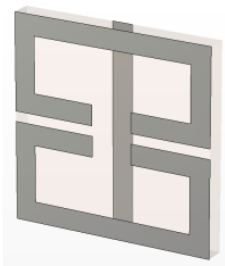

Axially symmetric SRR

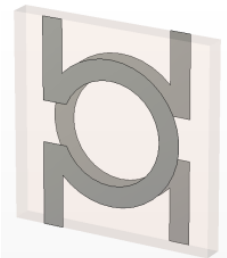

Omega SRR

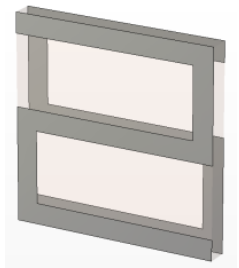

$\mathrm{S}$ ring

Figure 2. Overview of Split-Ring Resonators.

It has been demonstrated that periodic arrays of SRRs or other unit cell structures (i.e. single-ring or multi-ring SRRs, SRRs with multiple gaps, broadside-coupled SRRs, spiral resonators, S-shaped or omega-shaped resonators) may also display dipole-type resonances in addition to the LC-type resonances (Chen, Ran, Huangfu, Zhang \& Chen, 2004; Baena, Marques, Medina \& Martel, 2004). A feasible approach to design metamaterials with multiple and closely located resonance frequencies is to use a proper super-cell topology that is composed of two or more slightly different unit cells as explained in contributions from Kwon, Yuan Ekmekci and Zhu (Kwon, Werner, Kildishev \& Schalev, 2007; 
Yuan et al. 2008; Ekmekci, Topalli, Akin \& Turhan-Sayan, 2009; Zhu, Ma, Li \& Liang, 2010). Alternatively, a compact unit-cell structure called single-loop resonator (SLR) has been recently demonstrated by Ekmekci and Turhan-Sayan (Ekmekci \& Turhan-Sayan, 2010) with three resonance frequencies in X-band. The SLR was designed in square-shape by using a single conducting loop, which was specifically shaped to have two effective resonating gaps. While two distinct MNG regions resulted from the first two magnetic resonances of this highly symmetric SLR topology, the third (electrical) resonance was not strong enough to generate a further ENG band. Therefore, the suggested SLR structure was reported to be useful as a double-band magnetic resonator. In 2011 Yurduseven et at. presented a triple-band metamaterial array of triangular-shaped SLR unit cells (Yurdusen, Yilmaz \& Turhan-Sayan, 2011). This lattice shows four closely located resonances within the frequency range 3.3 - 5.1 GHz that leads to two distinctive MNG regions and one fully developed ENG region. This unit cell structure is made of a single loop of conducting strip printed over a low-loss dielectric substrate.

Chiral metamaterials (CMMs) have attracted vivid research activity due to their interesting negative refraction properties. In fact, CMMs are metamaterials made of unit cells without any mirror symmetry. This results in the breaking of the degeneracy between two circularly polarized waves, right circularly polarized (RCP) and left circularly polarized (LCP), that causes the difference of refractive index of RCP and LCP waves. Assuming a time-harmonic field $\left(e^{-j \omega t}\right)$, the constitutive relations of an isotropic and homogeneous chiral medium are as follows:

$$
\left(\begin{array}{l}
D \\
B
\end{array}\right)=\left(\begin{array}{cc}
\varepsilon_{0} \varepsilon & j \frac{k}{c} \\
-j \frac{k}{c} & \mu_{0} \mu
\end{array}\right)\left(\begin{array}{l}
E \\
H
\end{array}\right)
$$

where, $\varepsilon, \mu, c$, and $k$ are the relative permittivity, relative permeability, the speed of light in free space, and the chirality parameter, respectively. The refractive indices for RCP and LCP waves are expressed as $n \pm=n \pm k$ when $\mathrm{n}=\sqrt{ } \varepsilon \mu$. Generally, provided a large enough chirality, the negativity of $n+$ or $n$ - can be achieved as shown by Tretyakov (Tretyakov, Nefedov, Sihvola, Maslovski \& Simovski, 2003). On the basis of this principle, some CMM structures have been suggested by Plum et al. (Plum et al. 2009) such as twisted rosettes, twisted crosses by Zhou et al. (Zhou et al. 2009), twisted U-SRRs by Li et al. (Li, Zhao, Koschny, Kafasaki \& Soukoulis, 2011), double-layer CMM structure by Wu et al. (Wu, Zhang \& Zhong, 2010), L-shaped design by Li et al. (Li, Yang \& Dong, 2011), and conjugated gammadion by Zhao et al. (Zhao, Zhang, Zhou, Koschny \& Soukoulis, 2011), consisting of multiple layers exhibiting pronounced optical activity and a negative refractive index in an effective frequency band lower than $10 \mathrm{GHz}$. In 2012 Zafiri et al. (Zafiri, Soleimani \& Nayyeri, 2012) proposed a dual-band CMM structure with negative refractive index and interesting engineering. Due to its dual-band behavior, this structure is versatile for numerous applications such as dual-band ultrathin polarization devices, dual-band microwave absorbers, dual-band focusing, and gain enhancement and axial-ratio improvement of dual-band circularly polarized antennas. In 2012 Zhong et al. (Zhong et al. 2012) presented a new type of dual-band negative permittivity metamaterial based on a cross circular loop resonator (CCLR) with shorting stubs. The proposed metamaterial exhibits two typical $\varepsilon$-negative frequency bands, and the frequencies of both the two bands can be conveniently controlled by adjusting the positions of shorting stubs. On the basis of present printed circuit board (PCB) techniques, a left-handed media can be easily realized by a transmission line loaded with these resonators. Among them, the one utilized to generate a magnetic response is the SRR or open SRR (OSRR), whose fundamental resonance leads to a response to the magnetic field characterized by dispersive permeability as explained by Martín and Martel (Martín, Bonache, Falcone, Sorolla \& Marqués, 2003; Martel et al., 2004). Applying Babinet's principle, complementary SRR (CSRR) and open CSRR (OCSRR) were introduced by Falcone, Bonache and Vélez to design metamaterials with negative effective permittivity (Falcone et al. 2004; Bonache, M. Gil, I. Gil, J. García-García \& Martín, 2006; Vélez et al., 2009). Another approach was suggested by Schurig et al. to obtain electrical response makes use of the electric-field-coupled resonator (ELC) with a fundamental mode that couples strongly to the electric field (Schurig, Mock \& Smith, 2006).

In 2012 a new technique based on the concept of Stepped-Impedance Resonators (SIR) was introduced by Zarghooni (Zarghooni \& Denidni, 2012) for the miniaturization of a Double Split-ring Resonator (DSR) unit-cell. Stepped-Impedance Resonators (SIRs) have been used in the fields of band-pass filters and duplexers because of their interesting characteristics in terms of harmonic suppression and also because of their smaller length compared to quarter-wave transformers. This unit-cell can be used in a small-sized planar superstrate structure for gain enhancement of patch antennas.

\section{Metamaterial-Inspired Microwave Components}

The possibility of taking advantage of the unusual properties of the so-called metamaterial technology has led to an impressive research activity aimed at reducing the size and improving the performances of phase shifters, baluns, 
couplers, power dividers, and filters. A broad overview is given by Marques et al. (Marques, Martín \& Sorolla). The underlying idea for such improvement is the achievement of both negative permittivity and permeability over a certain frequency range by suitable adjustment of the dispersion characteristics.

At microwave frequencies, a medium that supports backward wave propagation can be easily fabricated from a transmission line loaded with split-ring resonators (SRRs) and/or reactive elements. These loading elements enable the efficient control of the response characteristics by simply adjusting size and position of the unit-cell. These structures generally incorporate resonant particles such SRRs, open SRRs (OSRRs), and their dual Babinet's counterparts (i.e. complementary split-ring resonators (CSRRs) or open CSRRs). Due to the resonant nature of these transmission lines, special efforts have been dedicated to the synthesis of artificial lines that display wider bandwidths. A broadband response can be obtained when the right- and left-handed behavior coincide. This effect is known as the balanced composite right/left-handed (CRLH) response. Generally, this kind of periodically loaded line has compact dimensions as a consequence of the electrically small size of the resonators used. It also yields low insertion loss and broad fractional bandwidth. However, the transmission line usually requires several unit-cells (typically between four and five) in order to obtain acceptable selectivity.

Distributed loading elements are employed in order to provide an improved control of the transmission line response. In general, inter-digital capacitors and shunt open-ended stubs are adopted. The stubs act as extra capacitors. In 2011 A. L. Borja et al. presented a unit-cell with enhanced bandwidth and compact dimensions (A. L. Borja, Belenguer, Cascón, Esteban \& V. E. Borja, 2011). The use of split-ring resonators together with metallic shunt strips provides negative permeability and permittivity, respectively. Similarly, the employment of additional series and shunt capacitances allow the control of both left- and right-handed bands, resulting in a balanced composite transmission line. This configuration exhibits a wide bandpass response as a result of this balance between advance and delay phase offsets.

An innovative application of metamaterial was introduced by Katko et al. (Katko, Hawkes, Barret \& Cummer, 2011). A sheet of nonlinear metamaterials was proposed for the realization of an RF limiter. In particular, complementary electric inductive-capacitive resonators loaded with nonlinear $\mathrm{p}-\mathrm{i}-\mathrm{n}$ diodes were combined to act as RF limiter unit-cells.

Nonlinear metamaterials also provide an attractive alternative to implement a shielding coating to suppress surface currents in Electromagnetic Compatibility (EC) problems as explained by Sievenpiper (Sievenpiper, 2011). A periodic arrangement of electrically small unit-cells behaves as an effective medium, whose properties depend on the circuit elements included in each unit-cell. By adding nonlinear devices such as diodes, an additional degree of freedom (that is not achievable with linear materials) can be obtained. The incoming wave can be converted into a different frequency, or a different mode, so to allow alternative absorption mechanisms.

\section{Antenna Applications}

The artificial electric and magnetic properties of metamaterials can be exploited to enhance radiation properties of antennas. Specifically, metamaterials have been employed to manipulate the radiation properties of antennas. Electromagnetic BandGap (EBG) cells distributed around the antenna have been used to increase the gain performance on sub-optimum ground plane by reducing the effects of surface waves. Ruvio and Ammann proposed a radial EBG-cell layout (Ruvio, Bao \& Ammann, 2009)as an alternative to conventional Cartesian solutions to enable more flexible design and better circular polarization features (Fig. 2). More recent designs include metamaterial-coated electrically small antennas investigated by Ziolkowski (Ziolkowski \& Erentok, 2006), transmission line (TL) metamaterial-enabled leaky-wave antennas designed by Liu et al. (Liu, Caloz \& Itoh, 2002), broadband negligible-loss metaliners for horn antennas that support low sidelobes and low cross polarization introduced by Lier et al. (Lier, Werner, Scarborough, Wu \& Bossard, 2011), metamaterial lenses for broadband highly directive multibeam antennas proposed by Zhou et al. (Zhou, Zhang \& Xin, 2010), high-gain conformal antennas prototyped by Jiang et al. (Jiang, Gregory \& Werner, 2011), and many others. Several metamaterial-based low-profile antennas have been successfully proposed by Qureshi, Erentok, Antoniades and Kokkinos that adopt metamaterial-like resonator structures placed close to the radiating structure (Qureshi, Antoniades \& Eleftheriades, 2005; Erentok \& Ziolkowski, 2008; Kokkinos \& Feresidis, 2009). The operating principle behind this approach is that the radiating element is sensitive to the presence of the resonator due to coupling. This alters the antenna's radiation characteristics by effectively acting as a "metamaterial shell". 

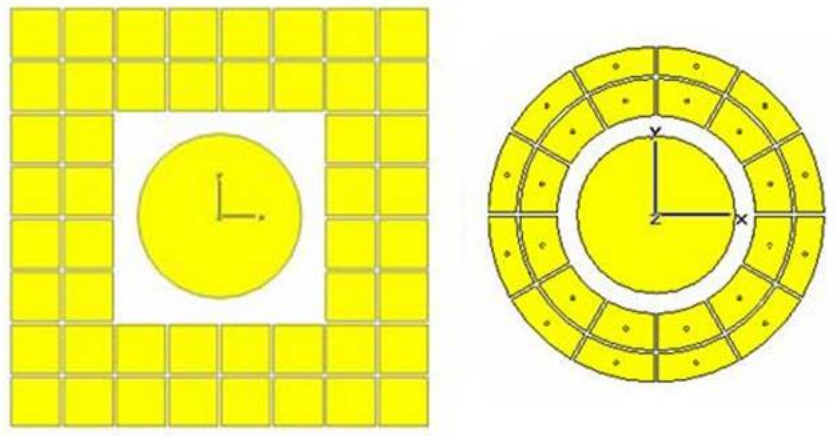

Figure 2. EBG cells arranged in Cartesian and radial layout around a patch antenna.

In 2011 Ntaikos proposed an electrically small planar monopole antenna in combination with a single SRR unit-cell, in such a way to create a composite compact planar antenna structure with an enhanced bandwidth and also multiband (WiMAX) operation (Ntaikos, Bourgis \& Yioultsis, 2011). The presence of the rings significantly shifts downwards the operation frequency.

A new type of compact flexible anisotropic metamaterial coating is proposed by Jiang (Jiang, Gregory \& Werner, 2011), which greatly enhances the impedance bandwidth of a quarter-wave monopole to over an octave. The metamaterial coating shows a high effective permittivity for the tensor component oriented along the direction of the monopole. Moreover, the coating produces an additional higher frequency resonance, which enables a similar current distribution on the monopole to that of the fundamental mode due to the metamaterial's broadband anisotropic behavior.

Recently, the anisotropic zero-index metamaterials (ZIM) have been proposed by Ma (Ma, Wang, Chen \& Ong, 2009). Compared to the isotropic ZIM, the anisotropic ZIM presents only one null component of the permittivity or permeability. These metamaterials have high efficiency to radiate and receive electromagnetic waves due to the improved impedance match. In 2011 Zhou and Cui proposed interesting features of anisotropic ZIM to develop a high-directivity Vivaldi antenna (Zhou \& Cui, 2011). The anisotropic ZIM structures can be embedded into the original Vivaldi antenna smoothly and easily with consequent enhanced directivity and gain in the bandwidth of anisotropic ZIM.

The basic working principle of Leaky-wave antennas (LWAs) is based on a wave propagating along a guiding structure and gradually leaking out a small amount of energy in the form of coherent radiation. Its advantages are the ability to offer sharp directional beams in a frequency scanning over a large bandwidth as explained by Tamir et al. (Tamir, Collin \& Zucker, 1969). There are two different basic types of LWAs: uniform (or quasi-uniform) and periodic. In uniform LWAs, the guiding structure is uniform along its length. In this case, the structure supports a wave that is fast compared to free space radiation. A quasi-uniform LWA operates in a similar way as does a uniform one, except that a periodic structure is used to guide the wave. However, the period is much smaller than the wavelength, and the periodicity does not play an active role in the radiation process. In periodic LWAs, the guiding structure supports slow waves with respect to free space. However, the periodic modulation of the guiding structure generates infinite space harmonics, some of these may be fast, hence providing leaky-wave radiation. Specifically, planar LWAs have recently attracted much attention due to their structural simplicity, easy fabrication, and integration with other planar components. The interest in printed LWAs has increased significantly, mainly due to the development of metamaterial transmission lines. Over the past years, metamaterial transmission lines have been proposed as backward, forward, or backward-forward frequency scanning LWAs in particular by Grbic and Chi (Grbic \& Eleftheriades, 2002; Chi \& Chen, 2013). A very interesting property of these leaky-wave antennas is their capability to operate in the fundamental mode. This avoids the need for a complicated and costly feeding network, as typically used in a phased array. Moreover, most of these antennas can radiate at broadside. Despite the fact that these fundamental modes are fast in a certain region of the dispersion diagram, a cancellation of the leaky-wave radiation occurs when open planar waveguides such as microstrip, coplanar waveguide (CPW), and coplanar strip (CPS) lines are used to design LWAs, due to the presence of antiparallel currents. Such antennas are usually considered as antenna arrays in which radiation is mainly due to periodically loaded radiating elements, which are excited by the fundamental propagating mode.

Due to their peculiar abilities to control and manipulate light, gradient index (GRIN) media have been widely used to realize several microwave devices as proposed by Smith (Smith, Mock, Starr \& Schuring, 2005). GRIN lenses have been proposed as alternatives to conventional technology, where refractive index is varied throughout the lens instead of relying on the interfaces of the dielectric material to control the light flow. Metamaterials have suitable physical properties to realize such structures. It has been lately shown by Cheng et al. (Cheng, Ma, \& Cui, 2009) that a planar medium composed of metallic inclusions on a printed circuit board can satisfy the refractive index profile of a Luneburg 
lens given by the relation

$$
n(r)=\sqrt{2-(r / R)^{2}}
$$

with $0 \leqslant r \leqslant R$, where $R$ is the radius of the lens and $r$ is the distance from any point to the lens center. It has been reported that a point source on the surface of such planar 2-D lenses is transformed into a collimated beam on its diametrically opposite side. In 2012 Dhouibi et al. realized the combination of a compact planar feeding structure with a substrate-integrated GRIN material assimilating a small electric size Luneburg lens (Dhouibi, Burokur, de Lustrac \& Priou, 2012). The gradient index of the Luneburg lens is achieved by Liu et al. through a two-dimensional array of waveguided units of complementary closed-ring (CCR) resonators (Liu, Cheng, Chin, Mock, Cui \& Smith, 2009).

\section{Imaging}

DNG metamaterials have attracted strong attention also in terms of imaging unique characteristics. Novel properties of metamaterial-based lenses have been proposed by Schurig and Smith in 2004 (Schurig \& Smith, 2004). Fig. 3 very simply describes the geometry of the scattering of a wave obliquetely incident upon a "double-negative" interface compared to a conventional "double-positive" one. The electromagnetic radiation from an isotropic line source located at one of the two focal lines of an elliptic cylinder made of DNG metamaterial was studied by Uslenghi in the frequency domain and in the optical limit (Liang \& Uslenghi, 2006). The permittivity and permeability of the metamaterial are assumed to be real, negative, and of opposite value to the corresponding parameters in the space surrounding the cylinder. Thus, the refractive index of the DNG cylinder is real, negative, and the opposite of the refractive index of the surrounding medium, whereas the intrinsic impedances of the two media have the same value. The DNG lenses have a line source located inside the lens, but the generated optical pattern appears to originate from a virtual line source located elsewhere.

Recently, there has been growing interest in metamaterial and plasmonic cloaking from Engheta and Pendry due to their intriguing properties and fascinating applications, including not only invisibility and camouflaging, but also non-invasive probing, imaging, cloaked sensing, and low-interference communications (Alù \& Engheta, 2008; Pendry, Schurig \& Smith, 2006).

However, the practical realization of cloaks is hindered by technological limitations, in particular when trying to reproduce specific profiles of bulk anisotropy and inhomogeneity for the effective permittivity $\overline{\overline{\varepsilon_{e f f}}}$ tensor and permeability $\overline{\overline{\mu_{e f f}}}$, as required in transformation-based cloaking devices. In addition, a thickness comparable to the region to be cloaked is usually necessary to achieve effective metamaterial bulk properties, a condition that clashes with the desire to keep small the total size of the cloaked region.

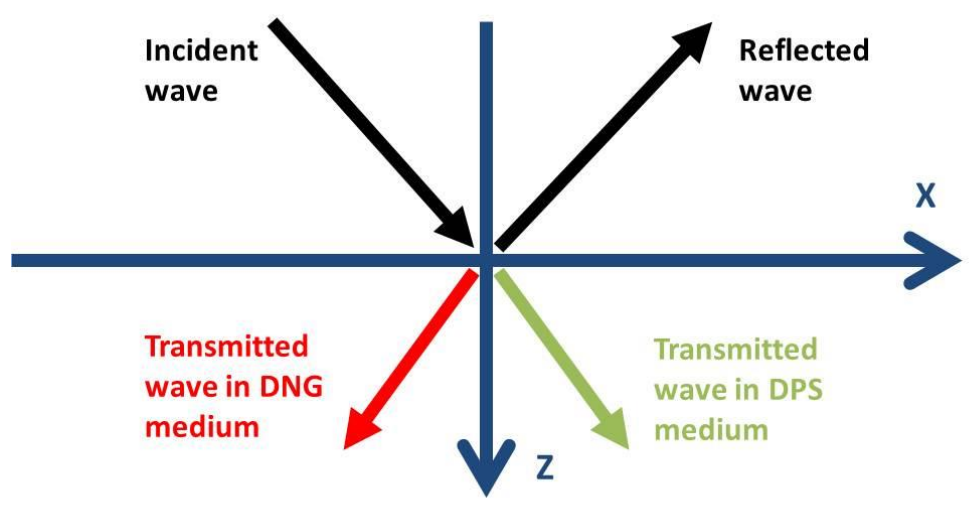

Figure 3. Scattered wave when obliquetely incident upon a DPS-DNG interface.

\section{Fabrication}

The growing interest in the electromagnetic properties of periodically structured materials was initiated by Yablanovitch and John in the late 1980's. 3-D periodic structures were originally obtained by mechanically drilling holes into dielectric material. The periodicity of the holes in such lattice structure generated a bandgap where propagation is impeded in any direction. Since then more sophisticated metallo-dielectric structures were proposed in the literature. This advancement also triggered the development of integration techniques such as stacking printed modules or 
growing lattice layer by layer for higher frequencies. Given the large frequency range where metamaterials find applications, their fabrication methods span from the simple mechanical shaping methods at lower frequencies to sophisticated photoligraphic schemes for the near-infrared spectrum region. Three characteristics determine the suitability of a particular integration technique:

- the scale of the metamaterial structure;

- the material used;

- the lattice pattern.

The dedicated literature suggests as a rule of thumb that the period of the metamaterial structure is a fraction of the free-space wavelength at the centre of the operating bandwidth. This corresponds to challenging fabrication issues for frequencies above $300 \mathrm{GHz}$. Further challenges need to be considered when a high degree of accuracy is required. A minimum fabrication tolerance must be taken into account for each integration procedure. In this framework it is appropriate to distinguish between random and systematic errors in the periodic lattice. A repeated difference in the structural period by the same amount from the designed vaue might cause an unacceptable frequency shift.

\section{Conclusions}

Metamaterials are a large family of metallo-dielectric structures with specific electromagnetic properties. By varying periodicity and/or LC contribution in each elementary cell, different combinations of electric properties can be derived (ENG, MNG and DNG). Such versatility is exploited in several conventional applications (antennas, filters, delay-lines, etc.) but it also suggested new ones like cloaking. Strong research efforts were dedicated on realization techniques that mainly aim to miniaturization and broad angular/bandwidth response. Several features make manufactured metamaterials limited or different than ideal references. These are mostly due to the truncation of the periodicity and a non-omnidirectional behavior.

Applications of metamaterials are still under development although at lower pace compared to 10 years ago. From an engineering perspective, metamaterial-enhanced antenna design is mature for practical purposes and commercial outcomes especially in defense applications where size-constraints can be largely compromised. In other engineering sectors the gap between manufacturing outcomes and expected behavior is still large and this discourages short-term commercial initiatives.

\section{Acknowledgements}

This work was supported by POR Campania FSE 2007/2013-"MASTRI".

\section{References}

Alù, A., \& Engheta, N. (2008). Plasmonic and metamaterial cloaking: Physical mechanisms and potentials. J. Opt. A, Pure Appl. Opt., 10(9), 093002. http://dx.doi.org/10.1088/1464-4258/10/9/093002.

Antoniades, M. A., \& Eleftheriades, G. V. (2009). A broadband dual-mode monopole antenna using NRI-TL metamaterial loading. IEEE Antennas Wireless Propag. Lett., 8, 258-261. http://dx.doi.org/10.1109/LAWP.2009.2014402.

Baena, J. D., Marques, J. D., Medina, F., \& Martel, J. (2004). Artificial magnetic metamaterial design by using spiral resonators. Phys. Rev. B, 69, 014402(1)-014402(5). http://dx.doi.org/10.1103/PhysRevB.69.014402.

Bonache, J., Gil, M., Gil, I., García-García, J., \& Martín, F. (2006). On the electrical characteristics of complementary metamaterial resonators. IEEE Microw. Wireless Compon. Lett., 16(10), 543-545. http://dx.doi.org/10.1109/LMWC.2006.882400.

Borja, A. L., Belenguer, A., Cascón, J., Esteban, H., \& Boria, V. E. (2011). Wideband Passband Transmission Line Based on Metamaterial-Inspired CPW Balanced Cells. IEEE Ant. Wir. Propag. Letters, 10, 1421-1424. http://dx.doi.org/10.1109/LAWP.2011.2178385.

Chen, H., Ran, L., Huangfu, J., Zhang, X., \& Chen, K. (2004). Left-handed materials composed of only S-shaped resonators. Phys. Rev. E, 70, 057605(1)-057605(4). http://dx.doi.org/10.1103/PhysRevE.70.057605.

Cheng, Q., Ma, H. F., \& Cui, T. J. (2009). Broadband planar Luneburg lens based on complementary metamaterials. Appl. Phys. Lett., 95(18), 181901. http://dx.doi.org/10.1063/1.3257375.

Chi Y., \& Chen, F. (2013). CRLH leaky-wave antenna based on ACPS technology with $180^{\circ}$ horizontal plane scanning capability. IEEE Trans. Antennas Propag., 61(2), 571-577. http://dx.doi.org/10.1109/TAP.2012.2224839.

Dhouibi, A., Burokur, S. N., de Lustrac, A., \& Priou, A. (2012). Compact Metamaterial-Based Substrate-Integrated Luneburg Lens Antenna. IEEE Ant. Wir. Propag. Letters, 11, 1504-1507. http://dx.doi.org/10.1109/LAWP.2012.2233191. 
Ekmekci, E., \& Turhan-Sayan, G. (2010). Single loop resonator: Dual-band magnetic metamaterial structure. Electron. Lett., 46(5), 324-325. http://dx.doi.org/10.1049/el.2010.3027.

Ekmekci, E., Topalli, K., Akin, T., \& Turhan-Sayan, G. (2009). A tunable multi-band metamaterial design using micro-split SRR structures. Opt. Exp., 17(18), 16046-16058. http://dx.doi.org/10.1364/OE.17.016046.

Erentok A., \& Ziolkowski, R. W. (2008). Metamaterial-inspired efficient electrically small antennas. IEEE Trans. Antennas Propag., 56(3), 691-707. http://dx.doi.org/10.1109/TAP.2008.916949.

Falcone, F., Lopetegi, T., Laso, M. A. G., Baena, J. D., Bonache, J., Beruete, M., Marqués, R., Martín, F., \& Sorolla, M. (2004). Babinet principle applied to the design of metasurfaces and metamaterials. Phys. Rev. Lett., 93(19), 197401. http://dx.doi.org/10.1103/PhysRevLett.93.197401.

Grbic A., \& Eleftheriades, G. V. (2002). Leaky CPW-based slot antenna arrays for millimeter-wave applications. IEEE Trans. Antennas Propag., 50(11), 1494-1504. http://dx.doi.org/10.1109/TAP.2002.804259.

Hand T. H., \& Cummer, S. A. (2009). Controllable magnetic metamaterial using digitally addressable split-ring resonators. IEEE Antennas Wireless Propag. Lett., 8, 262-265. http://dx.doi.org/10.1109/LAWP.2009.2012879.

Holloway, C. L., Kuester, E. F., Baker-Jarvis, J., \& Kabos, P. (2003). A double negative (DNG) composite medium composed of magnetodielectric spherical particles embedded in a matrix. IEEE Trans. Antennas Propag., 51(10), 2596-2603. http://dx.doi.org/10.1109/TAP.2003.817563.

Jiang, Z. H., Gregory, M. D., \& Werner, D. H. (2011). A Broadband Monopole Antenna Enabled by an Ultrathin Anisotropic Metamaterial Coating. IEEE Ant. Wir. Propag. Letters, 10, 1543-1546. http://dx.doi.org/10.1109/LAWP.2011.2180503.

Jiang, Z. H., Gregory, M. D., \& Werner, D. H. (2011). Experimental demonstration of a broadband transformation optics lens for highly directive multibeam emission. Phys. Rev. B, 84, 165111-1-165111-6. http://dx.doi.org/10.1103/PhysRevB.84.165111.

Katko, A. R., Hawkes, A. M., Barrett, J. P., \& Cummer, S. A. (2011). RF Limiter Metamaterial Using p-i-n Diodes. IEEE Ant. Wir. Propag. Letters, 10, 1571-1574. http://dx.doi.org/10.1109/LAWP.2011.2182490.

Kokkinos T., \& Feresidis, A. P. (2009). Low-profile folded monopoles with embedded planar metamaterial phase-shifting lines. IEEE Trans. Antennas Propag., 57(10), 2997-3008. http://dx.doi.org/10.1109/TAP.2009.2028605.

Kwon, D. H., Werner, D. H., Kildishev, A. V., \& Schalev, V. M. (2007). Nearinfrared materials with dual-band negative-index characteristics. Opt. Exp., 15(4), 1647-1652. http://dx.doi.org/10.1364/OE.15.001647.

Lewin, L. (1947). The electrical constants of a material loaded with spherical particles. Proc. Inst. Elect. Eng., 94(12), 65-68.

Li, J., Yang, F.-Q., \& Dong, J.-F. (2011). Design and simulation of L-shaped chiral negative refractive index structure. Prog. Electromagn. Res., 116, 395-408. http://dx.doi.org/10.2528/PIER11032601.

Li, Z., Zhao, R., Koschny, T., Kafesaki, M., \& Soukoulis, C. M. (2010). Chiral metamaterials with negative refractive index based on four U” split ring resonators. Appl. Phys. Lett., 97, 081901. http://dx.doi.org/10.1063/1.3457448.

Liang J., \& Uslenghi, P. L. E. (2007). A paraboloidal lens made of double-negative material. Radio Sci., 42, RS6S14. http://dx.doi.org/10.1029/2007RS003701.

Lier, E., Werner, D. H., Scarborough, C. P., Wu, Q., \& Bossard, J. A. (2011). An octave-bandwidth negligible-loss radiofrequency metamaterial. Nature Mater., 10(3), 216-222. http://dx.doi.org/10.1038/nmat2950.

Liu, L., Caloz, C., \& Itoh, T. (2002). Dominant mode leaky-wave antenna with backfire-to-endfire scanning capability. Electron. Lett., 38(23), 1414-1416. http://dx.doi.org/10.1049/el:20020977.

Liu, R., Cheng, Q., Chin, J. Y., Mock, J. J., Cui, T. J., \& Smith, D. R. (2009). Broadband gradient index microwave quasi-optical elements based on non-resonant metamaterials. Opt. Express, 17(23), 21030-21041. http://dx.doi.org/10.1364/OE.17.021030.

Mandelshtam, L. I. (1950). Group velocity in a crystal lattice. Zhurnal Eksperimentalnoi i Teoreticheskoi Fiziki, 15, 476-478.

Marques, R., Martin, F., \& Sorolla, M. (2008). Metamaterials With Negative Parameters: Theory, Design and Microwave Applications. Hoboken, NJ: Wiley.

Martel, J., Marqués, R., Falcone, F., Baena, J. D., Medina, F., Martín, F., \& Sorolla, M. (2004). A new LC series element for compact bandpass filter design. IEEE Microw. Wireless Compon. Lett., 14(5), 210-212. 
http://dx.doi.org/10.1109/LMWC.2004.827836.

Martín, F., Bonache, J., Falcone, F., Sorolla, M., \& Marqués, R. (2003). Split-ring resonator-based left-handed coplanar waveguide. Appl. Phys. Lett., 83(22), 4652-4654. http://dx.doi.org/10.1063/1.1631392.

Ntaikos, D. K., Bourgis, N. K., \& Yioultsis, T. V. (2011). Metamaterial-Based Electrically Small Multiband Planar Monopole Antennas. IEEE Antennas Wireless Propag. Lett., 10, 963-966. http://dx.doi.org/10.1109/LAWP.2011.2167309.

Pendry, J. B., Holden, A. J., Robbins, D. J., \& Stewart, W. J. (1999). Magnetism from conductors and enhanced nonlinear phenomena. IEEE Trans. Microw. Theory Tech., 47(11), 2075-2084. http://dx.doi.org/10.1109/22.798002.

Pendry, J. B., Schurig, D., \& Smith, D. R. (2006). Controlling electromagnetic fields. Science, 312, 1780-1782. http://dx.doi.org/10.1126/science.1125907.

Plum, E., Zhou, J., Dong, J., Fedotov, V. A., Koschny, T., Soukoulis, C. M., \& Zheludev, N. I. (2009). Metamaterial with negative index due to chirality. Phys. Rev. B, 79, 035407. http://dx.doi.org/10.1103/PhysRevB.79.035407.

Qureshi, F., Antoniades, M. A., \& Eleftheriades, G. V. (2005). A compact and low-profile metamaterial ring antenna with vertical polarization. IEEE Antennas Wireless Propag. Lett., 4, 333-336. http://dx.doi.org/10.1109/LAWP.2005.857041.

Ruvio, G., Ammann, M. J., \& Bao, X. L. (2009). Radial EBG Cell Layout for GPS Patch Antennas. Electronics Letters, 45(13), 663-664. http://dx.doi.org/ 10.1049/el.2009.1145.

Schurig, D., Mock, J. J., \& Smith, D. R. (2006). Electric-field-coupled resonators for negative permittivity metamaterials. Appl. Phys. Lett., 88, 041109. http://dx.doi.org/10.1063/1.2166681.

Shurig D., \& Smith, D. R. (2004). Negative index lens aberrations. Phys. Rev. E, 70, 065601. http://dx.doi.org/10.1103/PhysRevE.70.065601.

Sievenpiper, D. F. (2011). Nonlinear Grounded Metasurfaces for Suppression of High-Power Pulsed RF Currents. IEEE Ant. Wir. Propag. Letters, 10, 1516-1519. http://dx.doi.org/10.1109/LAWP.2011.2182593.

Silin, R. A. (1959). Waveguiding properties of two-dimensional periodical slow-wave systems. Voprosy Radioelektroniki, Elektronika, 4, 11-33.

Sivukhin, D. V. (1957). The energy of electromagnetic waves in dispersive media. Opt. Spektrosk., 3, 308-312.

Smith, D. R., Mock, J. J., Starr, A. F., \& Schuring, D. (2005). Gradient index metamaterials. Phys. Rev. E, 71(3). http://dx.doi.org/10.1103/PhysRevE.71.036609.

Smith, D. R., Padilla, W. J., Vier, D. C., Nemat-Nasser, S. C., \& Schultz, S. (2000). Composite medium with simultaneously negative permeability and permittivity. Phys. Rev. Lett., 84(18), 4184-4187. http://dx.doi.org/10.1103/PhysRevLett.84.4184.

Tamir T., Collin R. E., \& Zucker, F. J. (1969). Antenna Theory, McGraw-Hill.

Tao, H., Padilla, W. J., Zhang, X., \& Averitt, R. D. (2011). Recent progress in electromagnetic metamaterial devices for terahertz applications. IEEE J. Sel. Topics Quantum Electron., 17(1), 92-101. http://dx.doi.org/10.1109/JSTQE.2010.2047847.

Tretyakov, S., Nefedov, I., Sihvola, A., Maslovski, S., \& Simovski, C. (2003). Waves and energy in chiral nihility. J. Electromagn. Waves Appl., 17(5), 695-706. http://dx.doi.org/10.1163/156939303322226356.

Vélez, A., Aznar, F., Bonache, J., Velázquez-Ahumada, M. C., Martel, J., \& Martín, F. (2009). Open complementary split ring resonators (OCSRRs) and their application to wideband CPW band pass filters. IEEE Microw. Wireless Compon. Lett., 19(4), 197-199. http://dx.doi.org/10.1109/LMWC.2009.2015490.

Veselago, V. G. (1968). The electrodynamics of substances with simultaneously negative values of $\varepsilon$ and $\mu$. Sov. Phys. Usp., 10, 509-514. http://dx.doi.org/10.1070/PU1968v010n04ABEH003699.

Wu, Z., Zhang, B. Q., \& Zhong, S. (2010). A double-layer chiral metamaterial with negative index. J. Electromagn. Waves Appl., 24(7), 983-992. http://dx.doi.org/10.1163/156939310791285173.

Y. G. Ma, P. Wang, X. Chen, \& C. K. Ong. (2009). Near-field plane-wavelike beam emitting antenna fabricated by anisotropic metamaterial. Appl. Phys. Lett., 94, 044107. http://dx.doi.org/10.1063/1.3077128.

Yuan, Y., Bingham, C., Tyler, T., Palit, S., Hand, T. H., Padilla, W. J., Smith, D. R., Jokerst, N. M., \& Cummer, S. A. (2008). Dual-band planar electric metamaterial in the terahertz regime. Opt. Exp., 16(13), 9746-9752. http://dx.doi.org/10.1364/OE.16.009746. 
Yurduseven, O., Yilmaz, A.E., \& Turhan-Sayan, G. (2011). Triangular-Shaped Single-Loop Resonator: A Triple-Band Metamaterial With MNG and ENG Regions in S/C Bands. IEEE Ant. Wir. Propag. Letters, 10, 701-704. http://dx.doi.org/10.1109/LAWP.2011.2161742.

Zarghooni, B., \& Denidni, T. A. (2012). Design and simulation of a novel compact unitcell for DNG metamaterials based on stepped-impedance resonator technique. IEEE Ant. Propag. Soc. Intern. Symp. (APSURSI), 1-2. http://dx.doi.org/ 10.1109/APS.2012.6349409.

Zarifi, D., Soleimani, M., \& Nayyeri, V. (2012). Dual- and Multiband Chiral Metamaterial Structures With Strong Optical Activity and Negative Refraction Index. IEEE Ant. Wir. Propag. Letters, 11, 334-337. http://dx.doi.org/10.1109/LAWP.2012.2191261.

Zhao, R., Zhang, L., Zhou, J., Koschny, T., \& Soukoulis, C. M. (2011). Conjugated gammadion chiral metamaterial with uniaxial optical activity and negative refractive index. Phys. Rev. B, 83, 035105. http://dx.doi.org/10.1103/PhysRevB.83.035105.

Zhong, J., Huang, Y., Wen, G., Sun, H., Gordon, O., \& Zhu, W. (2012). Dual-Band Negative Permittivity Metamaterial Based on Cross Circular Loop Resonator With Shorting Stubs. IEEE Ant. Wir. Propag. Letters, 11, 803-806. http://dx.doi.org/10.1109/LAWP.2012.2208172.

Zhou B., \& Cui, T. J. (2011). Directivity Enhancement to Vivaldi Antennas Using Compactly Anisotropic Zero-Index Metamaterials. IEEE Ant. Wir. Propag. Letters, 10, 326-329. http://dx.doi.org/10.1109/LAWP.2011.2142170.

Zhou, J., Dong, J., Wang, B., Koschny, T., Kafesaki, M., \& Soukoulis, C. M. (2009). Negative refractive index due to chirality. Phys. Rev. B, 79, 121104. http://dx.doi.org/10.1103/PhysRevB.79.121104.

Zhou, R., Zhang, H., \& Xin, H. (2010). Metallic wire array as low-effective index of refraction medium for directive antenna application. IEEE Trans. Antennas Propag., 58(1), 79-87. http://dx.doi.org/10.1109/TAP.2009.2036282.

Zhu, C., Ma, J. J., Li, L., \& Liang, C. H. (2010). Multiresonant metamaterial based on asymmetric triangular electromagnetic resonators. IEEE Antennas Wireless Propag. Lett., 9, 99-102. http://dx.doi.org/10.1109/LAWP.2010.2042922.

Ziolkowski R. W., \& Erentok, A. (2006). Metamaterial-based efficient electrically small antennas. IEEE Trans. Antennas Propag., 54(7), 2113-2130. http://dx.doi.org/10.1109/TAP.2006.877179.

\section{$(\mathrm{cc}) \mathrm{BY}$}

This work is licensed under a Creative Commons Attribution 3.0 License. 